\title{
EL ÚLTIMO DISCURSO DEL PRESIDENTE MORA (Puntarenas, 17 de Setiembre de 1860)
}

\author{
President Mora's Last Address (Puntarenas, September 17, 1860)
}

\section{Oriester Abarca Hernández*}

\begin{abstract}
RESUMEN
Se presenta el hallazgo de un discurso, publicado en 1860 por The New York Times y el Panama Star \& Herald, que estos atribuyen a Juan Rafael Mora Porras. Afirman tales periódicos que este lo pronunció el 17 de setiembre de 1860, al arribar a la ciudad de Puntarenas. Se examinan algunos argumentos respecto a la plausibilidad de la hipótesis de la autenticidad del discurso de marras y se ofrece una reconstrucción, en castellano, a partir de las dos versiones en inglés.

Palabras clave: Juan Rafael Mora Porras, discurso, proclama, Puntarenas, 1860.
\end{abstract}

\section{ABSTRACT}

I examine the authenticity of a speech, published in 1860 by The New York Times and the Panama Star and Herald. These newspapers attribute it to Juan Rafael Mora Porras. They claim that Mora issued that proclamation on September 17, 1860, on his arrival in the city of Puntarenas. I feature some arguments about the hypothesis of the authenticity of that speech and propose a reconstruction of its Spanish original version. Key Words: Juan Rafael Mora Porras, speech, proclamation, Puntarenas, 1860. 


\section{Introducción}

Juan Rafael Mora Porras fue electo presidente de Costa Rica en 1849 y en 1853. Su más grande logro político fue haber declarado la guerra a William Walker y haberlo derrotado (1856-1857), poniendo así coto a las aventuras filibusteras del expansionismo estadounidense en Hispanoamérica.

Fue elegido para un tercer periodo en 1859 , pero el 14 de agosto de ese mismo año fue depuesto mediante un golpe de Estado. El 17 de setiembre de 1860 desembarcó en el puerto de Puntarenas, en el Pacífico costarricense, con la intención de retomar el poder por las armas. Según los periódicos Panama Star and Herald ("President Mora's Address", 1860, 27 de setiembre) y The New York Times (1860, 8 de octubre) al desembarcar pronunció un discurso, que publicaron traducido al inglés. El original en castellano al parecer no se conserva. El movimiento morista fue derrotado en pocos días y Mora Porras fue fusilado el 30 de setiembre de 1860 .

El cuerpo del artículo consta de tres partes. La primera expone las dos versiones del discurso. La segunda examina algunos argumentos que abonan la hipótesis de que el discurso es auténtico. La última sección presenta una propuesta de cómo pudo haber sido el texto original en castellano, a partir de las dos versiones, publicadas en inglés en 1860 por The New York Times y el Panama Star \& Herald; para ello se ha considerado el léxico y el estilo discursivo de Mora Porras.

\section{Las dos versiones publicadas en inglés}

Existen al menos dos versiones del discurso, publicadas en inglés, por periódicos diferentes. La primera apareció en la edición del 27 setiembre de 1860 del Panama Star and Herald. El Sacramento Daily Union, en su edición del 26 de octubre de 1860, también publicó una versión del discurso, pero aclaró que lo había tomado de otra fuente: "el Panama Star de 29 setiembre" de 1860 (claramente cometió una errata respecto a la fecha). La versión del Sacramento Daily Union difiere en unos pocos detalles respecto de su fuente. La segunda versión se publicó en The New York Times, en su edición del 8 de octubre de 1860.

Los textos publicados por el Panama Star and Herald y The New York Times son los siguientes: 
TABLA 1

Texto del discurso según las versiones publicadas por The New York Times y el Panama Star and Herald

Versión de The New York Times,
edición del 8 de octubre de 1860

Versión del Panama Star and Herald,

edición del 27 de setiembre de 1860

COSTA RICANS: When, in April last, I stated to you the causes that hindered me in aiding you to throw off the yoke of your oppressors, I could not foresee the magnitude of the sufferings which you now endure.

I never had the intention to abandon you: I asked you only to hope and to wait, and work for the felicity of the country; because I thought that time would reestablish peace among you without the necessity of my presence in the Republic.

Unfortunately, I deceived myself. More than one year has passed since the events of the 14th August. There has been, then, time enough to accomplish something for the good of the country had your rulers had the power or wish to do so. But, far from this, the past year has been to Costa Rica only a season of sorrow and tears. The people trampled upon and oppressed; our ports closed to commerce; the public treasury nearly bankrupt; -in fine, your territory sold, indirectly offered to filibusters. And all this without counting the discouragements and lack of confidence which weigh upon your labor and trouble your repose.

It is time that this shall end. Your repeated calls upon me, and the return of the filibuster WALKER, have decided myself and Gens. CAÑAS and MORA to sacrifice our tranquility for the independence and happiness of Costa Rica.

Soldiers! To arms! That the blood we shed in Nicaragua -at Santa Rosa, on the San Juan- may not have been spilled for nought. Come and unite with us, and fear nothing for the future.

It is not the desire for vengeance that directs our steps. Clemency is more conformable to my disposition, and I shall forget the errors of those who have been deceived into the service of the traitors. But from henceforth I shall be inexorable toward those who do not respond to the call of an agonized country, and shall cause all the rigor of the law to weigh upon those who render any sort of aid to the common enemy.

To arms! Costa Ricans! Hasten to unite with us, and to break your chains to the cry of "Viva Costa Rica!"

JUAN R. MORA.

Costa Ricans-When in April last I made known to you the reasons that prevented me from aiding you to cast off the yoke of your oppressors, I could not foresee the point which the magnitude of your sufferings today reaches.

I never intended to abandon you; I only asked you to wait and labor for the good of the country, because I thought that some would establish internal peace without the necessity of my presence in this republic.

Unfortunately I deceived myself. More than a year has passed since the events of the $14^{\text {th }}$ of August; they have had more than a year therefore, to do some good for the benefit of the country if they had been able or wished to. But far from it. The past year has only been for Costa Rica a page of tears and grief. The people punished and oppressed, the priests of Christ outraged and maltreated, the ports closed to commerce, the public treasury nearly made bankrupt -lastly, your country sold, offered indirectly to the filibusters; this without reckoning the general misery, the dismay and disconfidence that prevents you from dedicating yourself to labor and repose.

It will be necessary now that this be put an end to. Your frequent calls and the arrival in Central America of the filibuster Walker have decided me and Generals Cañas and Mora to sacrifice our tranquility for the independence and well-being of Costa Rica.

Soldiers-to arms! - that the blood you shed in Nicaragua, Santa Rosa, and the Rio San Juan, may not be without its fruits, join us, and you will have nothing to fear for the future.

It is nor vengeance that directs my steps, clemency is more in conformity with my feelings, and I will forget the errors of those who, deceived, served the traitors; but bear in mind, I will be inexorable towards those who from this day forth do no listen to the call of their agonized country, and I will cause all the vigor of the law to fall on those who assist or aid in any way the common enemy.

To arms, Costa Ricans! Hasten to join us, and to break your chains to the cry of VIVA Costa RicA.

Punta Arenas ${ }^{1}$, Sept. 17, 1860.- JuAN R. MoRA.

Fuentes: The New York Times (1860, 8 de octubre: 8), Panama Star and Herald (1860, 27 de setiembre). 


\section{Respecto a la autenticidad del discurso}

El discurso tiene sin duda muchas similitudes con otros documentos cuya autoría se atribuye a Mora Porras, tales como mensajes presidenciales, proclamas y discursos, pero la proclama que este suscribe en enero de 1860 en San Salvador (Mora Porras, El Presidente de la
República a los pueblos de Costa Rica, 1860), y la proclama de 29 de marzo de 1860, también suscrita en esa ciudad (Mora Porras, Juan Rafael Mora a los pueblos de Costa Rica, 1860) son los documentos a los que más se asemeja. Sin pretensión de ser exhaustivo, en la tabla 2 presento una comparación entre el texto que he fijado en castellano a partir de las dos versiones publicadas en inglés, arriba reproducidas, y otros documentos de Mora Porras.

Tabla 2

Comparación entre el discurso traducido y otros discursos, mensajes presidenciales y proclamas de Juan Rafael Mora Porras

Discurso de 17 de setiembre de 1860

$1^{\circ}$ Cuando en abril próximo pasado os hice saber las razones que me impidieron ayudaros a sacudir el yugo de vuestros opresores, no pude prever la magnitud que hoy alcanzan vuestros sufrimientos.
Fragmentos de otros discursos, mensajes presidenciales o proclamas de Juan Rafael Mora Porras

Cuando el 3 de agosto del año próximo pasado, ofrecí a vuestra consideración el cuadro que presentaba la República, y os hice en este mismo lugar una reseña de los sucesos que se habían desarrollado hasta aquella época, mi corazón se agitaba y conmovía por la incertidumbre del porvenir que esperaba a la República.

(Mensaje presidencial sobre el estado de la República en 1857, 6 de setiembre de 1857, inicio del primer párrafo)

Comentario:

La forma en que se inician ambos documentos es similar.

$2^{\circ}$ Nunca intenté abandonaros; solo os pedí esperar y No me culpéis pues, compatriotas, si me he fijado decididamente trabajar por el bien del país, porque pensé que el en este benévolo país. Culpad solo a las circunstancias y unidos tiempo restablecería la paz interna, sin que mi presencia fuera necesaria en esta República. todos trabajad sin descanso por la felicidad de nuestra patria. Este es el voto más ardiente de vuestro amigo y compatriota. (Proclama de 29 de marzo de 1860, párrafo final).

Comentario:

Mora Porras se excusa por su ausencia y pide al pueblo seguir trabajando por el bien (felicidad) del país.

$3^{\circ}$ Desafortunadamente, estaba equivocado. Más de Ya lo habéis palpado en cuatro meses que han tenido el Poder: un año ha transcurrido desde los hechos del 14 de nada han hecho sino es su propio negocio y perseguir a todas las agosto. Entonces, vuestros gobernantes han tenido clases de la sociedad...

tiempo suficiente para hacer algo en beneficio del (Proclama de San Salvador, enero de 1860, parte del quinto país, si hubieran sido capaces o lo hubieran querido. párrafo) 
Comentario:

Ambos textos coinciden en la idea de que en el tiempo que los golpistas habían ostentado el poder, no habían administrado en beneficio del país y que no habían hecho nada al respecto.

$4^{\circ}$ Pero lejos de ello, el pasado año solo ha sido Aquí no encontrarán jamás los invasores, partidos, espías, ni para Costa Rica una página de lágrimas y dolor. traidores. ¡Ay del nacional o extranjero que intentara seducir la El pueblo castigado y oprimido, los sacerdotes de Cristo ultrajados y maltratados, los puertos cerrados al comercio, el Tesoro Nacional casi en bancarrota; en fin, vuestro país vendido, ofrecido indirectamente a los filibusteros. Y todo esto sin considerar la miseria general, el desaliento y la desesperanza que hacen más pesado vuestro trabajo y perturban vuestro reposo. inocencia, fomentando discordias o vendernos!

(Primera proclama, 20 de noviembre de 1855, primera parte del párrafo final).

... ni el clero, ni la ancianidad, ni la juventud han estado seguros, todos han sufrido y sufren aún; vosotros lo sabéis mejor que yo.

(Proclama de San Salvador, enero de 1860, parte final del quinto párrafo)

Comentario:

Los textos se refieren a la venta del país, como una forma de traición y a los sufrimientos del pueblo y la mala gestión económica de la administración de Montealegre.

$\mathbf{5}^{\circ}$ Ha llegado ya el día de que esto se acabe. Vuestros He jurado sacrificar mi fortuna, mi reposo, mi vida, por el frecuentes llamamientos y el regreso a América buen nombre y por la independencia de mi patria. Cumpliré mi Central del filibustero Walker, nos han movido, a los generales Cañas y Mora y a mí, a sacrificar nuestra tranquilidad, por la independencia y el bienestar de Costa Rica.

juramento.

Si nuevos peligros nos llaman a la lid, cuento siempre con vosotros aquí, en la frontera, en todas partes.

(Alocución a las milicias de San José, 6 de julio de 1856, párrafo tercero y la parte inicial del cuarto)

Comentario:

Ambos textos aluden al sacrificio que hace Mora de su propia tranquilidad por la defensa del país y del llamado que las circunstancias o el pueblo le plantean para acudir a la lid.

$6^{\circ}$ ¡Soldados, a las armas! Que la sangre que ¿Pero, habrá concluido todo? No, compatriotas. La obra derramasteis en Nicaragua, Santa Rosa y el río empezada es menester terminarla; es forzoso que no quedemos San Juan no sea estéril. Y así venid a uniros con nosotros, y no tendréis nada qué temer del porvenir. expuestos a que un nuevo Walker vuelva a turbar nuestra paz, batallando por esclavizarnos; es preciso que tantos obstáculos vencidos, tantos sacrificios hechos, no sean estériles, y para ello es necesario continuarlos. Levantemos, pues, sobre el mismo río y con nuestras propias manos, un dique poderoso que contenga para hoy y para lo futuro ese torrente usurpador...

(Tercera proclama, 11 de enero de 1857 , primera parte del párrafo quinto)

Y así venid a uniros con el ejército libertador para que por segunda vez os deba su existencia esta patria querida.

(Proclama de San Salvador, enero de 1860, parte del segundo párrafo)

Comentario:

Coinciden los textos en que el precio pagado (la sangre derramada, los sacrificios hechos) no sean estériles respecto a continuar una lucha para lograr un mejor futuro. 
$7^{\circ} \quad$ No es el deseo de venganza lo que guía mis pasos; la clemencia se conforma mejor a mis sentimientos; así es que olvidaré los errores de aquellos que, engañados, prestaron sus servicios a los traidores; pero tened presente que seré inexorable con aquellos que, de hoy en adelante, no respondan al llamamiento de su agónico país, y haré que todo el rigor de la justicia caiga sobre aquellos que presten cualquier tipo de ayuda al enemigo común.
No me ha movido ninguna pasión innoble: ni el odio, ni el odio, ni el encono ni la venganza existe en mi alma: vuestra noble abnegación, vuestro patriotismo, vuestra lealtad y vuestro sufrimiento han sido la palanca que levantó mi espíritu, así es que habrá perdón general para todos, menos para los traidores Salazar, Rodríguez, Pacheco y Zaret, porque perdonar a tales malvados sería renunciar para siempre del orden y de la estabilidad de las cosas; sería ofender a la moral pública y sistematizar la indisciplina.

(Proclama de San Salvador, enero de 1860, primera parte del segundo párrafo)

Aquí no encontrarán jamás los invasores, partidos, espías, ni traidores. ¡Ay del nacional o extranjero que intentara seducir la inocencia, fomentando discordias o vendernos! Aquí no encontrarán más que hermanos, verdaderos hermanos, resueltos irrevocablemente a defender la Patria como a la santa madre hasta el último de sus enemigos.

(Primera proclama, 20 de noviembre de 1855, párrafo final) Los filibusteros, relacionados con los ambiciosos enemigos de la patria, no cesan en sus maquinaciones; aquí mismo han pretendido emponzoñar nuestra ventura para dividirnos, lanzarnos a una lucha intestina, y cuando Costa Rica se presentara destrozada, manchada de sangre y de baldón, cubierta de cadáveres y ruinas; cuando la vieran convertida por medio de la falacia, de la calumnia, de la traición, de las revoluciones y de una guerra fratricida en otra infeliz Nicaragua, uncirla al carro de la fortuna de una horda de bandidos. No lo conseguirán. Si hasta ahora he sido indulgente, de hoy en más la inflexible espada de la ley caerá sobre sus frentes criminales.

(Alocución a las milicias de San José, 6 de julio de 1856, texto del párrafo tercero)

Dueños del río y del Gran Lago, puestos en relación con nuestros aliados, reducido Walker a Rivas y sus alrededores, a ser estrechado, atacado y abrasado, si es preciso, con los restos de la ciudad donde se encierra. He brindado el perdón a todos los que obcecadamente siguen su causa, si le abandonan. Sabremos vencer y perdonar.

(Tercera proclama, 11 de enero de 1857 , párrafo cuarto)

Todos los filibusteros, de cualquier nacionalidad a que pertenezcan, que sean aprehendidos con las armas en la mano, sufrirán inmediatamente el rigor de la ley siendo fusilados.

Los que, sin haber hecho armas contra la República, las depongan voluntariamente, deserten de sus filas y se presenten ante los jefes del ejército nacional, serán perdonados.

(Decreto de 27 de marzo de 1856)

... ha llegado ya el día de la expiación para los malvados que se sublevaron el 14 de agosto y para los que se obstinen en ayudarlos, de hoy en adelante.

(Proclama de San Salvador, enero de 1860, parte final del párrafo primero)

Abandonad nobles costarricenses a esos cuatro traidores, yo os lo aconsejo por vuestro propio bien; escuchad mi voz de clemencia si no queréis que más tarde caiga sobre vuestras cabezas la espada de la justicia.

(Proclama de San Salvador, enero de 1860, parte final del párrafo tercero) 
Comentario:

En distintos contextos y momentos, Mora promete ser severo con los enemigos y traidores, pero clemente con los que abandonen la causa de estos.

$\mathbf{8}^{\circ} \quad$ ¡A las armas, costarricenses! Apresuraos a uniros con nosotros y a romper vuestras cadenas al grito de ‘ $\mathrm{VIVA}$ Costa RicA'!

¡Compatriotas! ¡A las armas! Ha llegado el momento que os anuncié. Marchemos a Nicaragua a destruir esa falange impía que la ha reducido a la más oprobiosa esclavitud: marchemos a combatir por la libertad de nuestros hermanos.

A la lid, pues, costarricenses. Yo marcho al frente del Ejército Nacional. Yo que me regocijo al ver hoy vuestro noble entusiasmo, que me enorgullezco al llamaros mis hijos, quiero compartir siempre con vosotros el peligro y la gloria.

(Segunda proclama, $1^{\circ}$ de marzo de 1856 , párrafos inicial y cuarto)

Costarricenses: cuento para todo con vosotros. Con vuestro apoyo y la protección divina, nada habrá que me haga retroceder. Bendigamos a la Providencia que nos ampara, y al grito de ¡VIVA Costa Rica! marchemos SIEMPRE unidos adelante, con fe y constancia en el porvenir.

(Tercera proclama, 11 de enero de 1857, párrafo final)

¡A LAS ARMAS COSTARRICENSES!

(Proclama de San Salvador, enero de 1860, parte del párrafo primero)

Comentario:

Mora usa los mismos lemas en diferentes documentos.

Fuentes: Texto en castellano fijado por mí a partir de las versiones en inglés publicadas en Panama Star and Herald (1860) y The New York Times (1860). Para los textos conocidos de Mora se utiliza las versiones publicadas por Vargas Araya (2013: 368-388), Durán Luzio (2011) y Meléndez Chaverri (1968: 185). El decreto de 27 de marzo de 1856 se tomó de Montúfar (1887: 292-293).

Si el discurso publicado por The New York Times y Panama Star and Herald es apócrifo, su autor debió de conocer profundamente las ideas y el estilo discursivo de Mora Porras. De otra manera no se podrían explicar las similitudes con los mensajes presidenciales, las proclamas y otros discursos del ex presidente.

EN CUANTO A POR QUÉ NO CIRCULÓ EL DISCURSO EN EL MEDIO COSTARRICENSE, PODRÍA SUGERIRSE QUE LA COYUNTURA MISMA DE GUERRA CIVIL DA BASE PARA UNA HIPÓTESIS PLAUSIBLE. PUBliCAR EL DISCURSO PUDO SER INTERPRETADO POR MONTEALEGRE Y SUS SEGUIDORES COMO TRAICIÓN O SEDICIÓN. DE HECHO, SOLO PODÍA SER DEL INTERÉS DE LOS MORISTAS PUBLICARLO, PERO SU MOVIMIENTO FUE DERROTADO EN POCOS DÍAS. SE SABE QUE LA ADMINISTRACIÓN DE MONTEALEGRE
CONOCIÓ EL DISCURSO EN LA VERSIÓN PUBLICADA POR EL Panama Star and Herald, pero no lo tradujo ni lo publicó; tampoco hizo alusión a él, a pesar de que dedicó espacio en la Gaceta Oficial de Costa Rica (13 de octubre de 1860: 3-4) a refutar las manifestaciones de aquel periódico que acompañaron la publicación del discurso de marras (Díaz Arias, 2014: 10).

No existe duda de que Mora Porras pronunció un discurso o proclama al desembarcar en Puntarenas el 17 de setiembre de 1860, pues incluso los gobernantes a los que quería derrocar así lo reconocieron:

El 14 en la noche dio Arancibia el grito de rebelión en Esparza, y después de reducir a prisión a las Autoridades del lugar, marchó como con cincuenta 
rebeldes hacia Puntarenas, que en la misma noche ocupó en nombre de D. Juan Rafael Mora...

Dos días después del atentado de Esparza, desembarcó en Puntarenas el pretendido Presidente de la República, en compañía de los ex generales Cañas y Mora, de su sobrino Argüello, y de unas pocas personas más. La proclama que expidió a su llegada, es un documento de refinada hipocresía, audaz, calumnioso y contradictorio en sentido y en ideas. (Gaceta Oficial de Costa Rica, 1860, 14 de diciembre: 4)

Otro punto que abona la hipótesis de que no se trata de un discurso apócrifo, es el hecho de que The New York Times declaró, en su edición del 8 de octubre de 1860, que fue su propio corresponsal, identificado como F. W. R., quien el 25 de setiembre de 1860, desde Panamá, remitió la información respecto al desembarco de Mora Porras, su recibimiento por parte de la población de Puntarenas y el discurso que pronunció. Esto sugiere que la fuente de The New York Times no es la misma que la del Panama Star and Herald, pues este tenía su propio corresponsal en la ciudad de Puntarenas (así lo afirmó el Daily Alta California, en The Tragedy - Execution of Ex-President Mora and General Canas, 1860).

CABE CONSIDERAR, TAMBIÉN, EL GRADO DE DETALLE CON QUE EL CORRESPONSAL DE The New York Times describió la llegada y la presencia de Mora Porras en la ciudad de Puntarenas. De modo que la fuente parece ser de primera mano. Según nuestra traducción de ese relato:

el día 14 de los corrientes [setiembre de 1860], el legítimo presidente de Costa Rica, Gen. J. R. MORA; su hermano, el Gen. JOAQUÍN MORA; su cuñado, el Gen. CAÑAS, quien fue su Secretario de Estado; el señor Aguilar ${ }^{2}$, y nueve seguidores y sirvientes, se embarcaron en La Libertad, El Salvador, en el vapor Columbus, de la Panama Railroad Company, habiendo tomado y pagado sus boletos para Panamá ${ }^{3}$. Un grupo tan grande de viajeros lleva, naturalmente, una cantidad considerable de equipaje y algunas cosas para hacer negocios; de modo que el Capitán LUDWIG no se sorprendió de encontrar las cubiertas cargadas de baúles y cajas; los primeros estaban todos etiquetados "para Panamá" y las segundas enviadas, de acuerdo con el plan, al mismo destino. El ex presidente, cuyos modales son, en general, extremadamente corteses y afables, parecía haber adquirido una cantidad adicional de cortesía y buen humor, de su saludable y placentera última ocupación como plantador de cafetos en El Salvador, y fue, los tres días que transcurrieron entre La Libertad y Puntarenas, el alma del pequeño grupo de pasajeros que iba en la cabina. La mañana del 17 de setiembre, el Capitán LUDWIG divisó Puntarenas desde la amura de babor y, tomando su catalejo, distinguió un asta en la orilla que hacía la señal usual que indica "vapor a la vista"; sobre el asta, una gran bandera ondeaba con la brisa de la mañana. Cuando se percató de aquel hecho, el presidente Mora exclamó: “¡Muy bien! ¡Esa es mi señal!” El buque ancló y fue rodeado, inmediatamente, por botes. En uno que estaba bajo el mando del Capitán de Puerto [el coronel Salvador Mora] ${ }^{4}$, el Gen. [Juan Rafael] Mora y su comitiva fueron invitados a dirigirse a tierra. Al llegar a la playa, el grupo entero fue rodeado por la población de Puntarenas; tal escena de abrazos y estrechamiento de manos rara vez se ha visto, aun en estos vivaces americanos tropicales. Al ingresar a la ciudad, vieron banderas ondeando en cada ventana y carteles de "¡Viva el presidente Mora!" en cada puerta. La población había tomado, dos días antes, las armas del gobierno y había depuesto a todos los oficiales del usurpador MONTEALEGRE, y desde entonces, vigilaba ansiosamente, a la espera del vapor que sabía traería a sus líderes preferidos, los MORA y CAÑAS.

MÁs de 200 HOMBres, REUNIDOS POR EL GRUPO QUE SE PRONUNCIABA, FUERON INMEDIATAMENTE DOTADOS DE ARMAS, Y SU NÚMERO FUE CRECIENDO RÁPIDAMENTE. LA GARGANTA DE ARENA DE SOLO UNAS POCAS VARAS DE ANCHO -QUE UNE LA CIUDAD CON EL ÚNICO CAMINO A SAN JOSÉ, LA CAPITAL-, AL TENER EL MAR A UN LADO Y UN ESTERO AL OTRO, FUE PUESTA EN ESTADO DE DEFENSA CON UNA BATERÍA DE SEIS CAÑONES, LOS CUALES SE UBICARON EN LA PARTE mÁs estrecha, La ANGostura. MORA SE ocupabA DE REALIZAR FUERTES PREPARATIVOS, YA FUESE PARA LA DEFENSA O PARA MARChar a SAN José, A 75 MILLAS DE DISTANCIA, SEGÚN LAS CIRCUNSTANCIAS LO REQUIRIESEN. Sin DUDA, PREVIAMENTE SE habíA ACORDADO QUE SE REALIZARÍAN LEVANTAMIENTOS DE la Población en San José, Cartago, Alajuela Y Heredia, LAS CIUdAdes MÁs POBladAS DEL INTERIOR. NO OBSTANTE, CUANDO EL COLUMBUS zarpó de Puntarenas la mañana Del 18 [DE SETIEMBRE DE 1860], SE IGNORABA EL ÉXITO QUE TALES LEVANTAMIENTOS PODRÍAN TENER, PUES DURANTE CUATRO DÍAS NO SE HABÍA TENIDO NINGUNA COMUNicación con San José. Pero el PREsidente MORA y la gente de Puntarenas esperaban LOGRAR EL ÉXITO, LO QUE INDICA QUE CONFIABAN 
EN SUS AMIGOS DEL INTERIOR, PORQUE SIN UNA EFICIENTE AYUdA DE PARTE DE ESA REGIÓN, MORA NO PODRÍA PASAR LOS DEFENDIBLES PUNTOS FUERTES de los Montes del Aguacate y La Garita. MONTEALEGRE PODRÍA FÁCILMENTE MANTENER ESAS POSICIONES, AUN CONTRA UN NÚMERO VEINTE VECES MAYOR, SI SUS SOLDADOS SE MANTIENEN LEALES Y LOS PUEBLOS EN SU RETAGUARDIA, APÁTICOS.

TAN PRONTO COMO LLEGÓ A TIERRA, EL PRESIDENTE MORA PRONUNCIÓ LA SIGUIENTE PROCLAMA: ... [SIGUE EL DISCURSO QUE SE REPRODUJO EN LA TABLA 1, COLUMna IZQuierda] (The New York Times, 1860, 8 de octubre: 8 )

Si nos atenemos a la narración del corresponsal de The New York Times, el desembarco de Mora Porras tuvo lugar en horas de la mañana y fue entonces cuando pronunció el discurso que nos ocupa.

Algunos DETALles DE LA NARRACiÓN SE CONFIRMAN CON OtRas FUeNTES. Por EJEMPlo, OTIS EN 1861 REPORTÓ DETALLES SOBRE EL VAPOR Columbus como una de las dos naves de la línea de vapores de Centroamérica de la Panama Railroad Company. Según Otis (1861: 149), el vapor Columbus tenía una capacidad de 460 toneladas y su capitán era J. W. Ludwig. Junto con el vapor Guatemala (con capacidad de 1.021 toneladas y cuyo capitán era J. M. Dow), formaba una línea quincenal, de acuerdo con la siguiente tabla de fechas aproximadas de partida y arribo a diferentes puertos de América Central:

TABLA 3.

Itinerario aproximado de los vapores Guatemala y Columbus 1861

\begin{tabular}{lcclcc}
\hline \multicolumn{1}{c}{ De } & Partida & \multicolumn{3}{c}{ Arribo } \\
\hline Panamá & 15 & Columbus & A & Guatemala & Columbus \\
\hline Puntarenas & 18 & 30 & Puntarenas & 17 & 2 \\
\hline Realejo & 20 & 5 & Realejo & 19 & 4 \\
\hline La Unión & 21 & 6 & La Libertad & 22 & 5 \\
\hline La Libertad & 22 & 7 & Acajutla & 22 & 7 \\
\hline Acajutla & 22 & 7 & San José & 23 & 7 \\
\hline San José & 25 & 10 & Acajutla & 25 & 8 \\
\hline Acajutla & 26 & 11 & La Libertad & 27 & 10 \\
\hline La Libertad & 27 & 12 & La Unión & 28 & 11 \\
\hline La Unión & 30 & 15 & Realejo & 30 & 13 \\
\hline Realejo & $1^{\circ}$ & 16 & Puntarenas & 2 & 17 \\
\hline Puntarenas & 3 & 18 & Panamá & 5 & 20 \\
\hline
\end{tabular}

Fuente: Otis (1861: 149). 
Como se expone en la Tabla 3, el Columbus arribaba a Puntarenas el día 17 de cada mes y partía de ese puerto el día siguiente (18 de cada mes), lo que concuerda con los datos aportados por la narración de The New York Times.

EL Sacramento Daily Union narró el desembarco de Mora Porras de la siguiente forma:

El día 15 de setiembre -o cerca de ese día- un grupo armado, dirigido por un tal Santander ${ }^{5}$ -revolucionario peruano, pero quien más recientemente ha figurado como actor de teatro-, previendo la llegada de Mora desde San Salvador en el vapor que se esperaba para el siguiente día, ingresó al cuartel, desarmó al centinela y tomó posesión sin oposición alguna. Mora, acompañado por su hermano y varios otros partidarios, llegó puntualmente en el Columbus y, al bajar a tierra, fue inmediatamente proclamado presidente de la República por el pueblo de Puntarenas. Mora y su comitiva salieron del vapor, subieron al bote de la Capitanía y se dirigieron a la orilla; pero en ese momento la marea estaba bajando con gran fuerza y el nivel del agua era muy bajo; en realidad, tanto que parecía imposible que el bote navegara. En vano los hombres remaban; en vano el patrón indio agotaba su vocabulario de elegantes exclamaciones; en vano el Capitán de Puerto suplicaba a los muchachos ${ }^{6}$ que se rompieran las espaldas por amor a Mora; finalmente se les obligó, a pesar de la ominosa apariencia del asunto, a levantar a su Excelencia y a subirlo a las espaldas de negros para llevarlo inmediatamente a tierra. Y así él [Mora Porras] hizo su entrada a Costa Rica. (End of the Revolution in Costa Rica, 1860: 1)

Esta versión puede corroborarse parcial e indirectamente por medio de un relato de Thomas Francis Meagher, quien desembarcó en Puntarenas el 11 de marzo de 1858. Al igual que Mora Porras, Meagher llegó en el Columbus, el cual describió como un "barco viejo a todas luces, al que han puesto una hélice". Pero lo más interesante es su relato acerca de la forma cómo, al estar la marea baja, hubo de alcanzar tierra firme:

Mirado desde el ardiente golfo de Nicoya, Puntarenas resulta hermoso, pero está bastante atrasado. No tiene malecón, ni muelle, ni embarcadero nuevo ni viejo, ni nada que se le parezca. Se baja a tierra en un bote, un bongo o un lanchón, como le venga a uno en gana o lo permita el bolsillo. Un bote cuesta un peso. A marea baja, las últimas cincuenta yardas del viaje hasta el pueblo, que son del cieno más pegajoso, tienen que hacerse a espaldas de un natural del país cuyas rodillas, según puedo atestiguarlo, no son de lo más firme cuando se les pone a prueba de 200 libras de carne irlandesa, una escopeta de dos cañones y unas botas de montar por añadidura. (Meagher, 1923: 5-6)

El relato de The New York Times concuerda con la descripción del Sacramento Daily Union en el sentido de que Mora Porras intentó llegar a tierra en el bote de la Capitanía de Puerto. A su vez el relato de este segundo periódico coincide con la narración de Meagher sobre la utilización de nativos para que llevaran sobre sus espaldas a los visitantes cuando la marea estaba baja. La detallada descripción que hace The New York Times parece entonces admisible, lo que sugiere que puede atribuirse a un testigo real, a alguien que presenció los hechos que narra.

TAMBIÉN ABONA LA HIPÓTESIS DE QUE EL DISCURSO DEL 17 DE SETIEMBRE DE 1860 ES AUTÉNTICO, EL HECHO DE QUE EXISTEN DOS VERSIONES EN INGLÉS, LAS CUALES SE SUPONE SON TRADUCCIONES DE UN MISMO ORIGINAL EN ESPAÑOL. AMBAS VERSIONES USAN DIFERENTES PALABRAS Y EXPRESIONES, PERO SUS CONTENIDOS COINCIDEN. EXISTE UNA deep structure (Chomsky, 1957) común a ellos.

\section{Algunos criterios sobre el discurso}

He consultado a algunos estudiosos de Mora Porras y han expresado sus criterios respecto al discurso en cuestión. Según Luko Hilje la hipótesis de que el discurso es auténtico tiene un alto grado de plausibilidad:

¿Quién y por qué habría intentado escribir un discurso así, sino don Juanito? Me parece que la versión original de él llegó a dos corresponsales diferentes, y cada uno la tradujo a su manera.

SE PUEDE HIPOTETIZAR QUE DON JUANITO REDACTÓ EL Discurso DURANTE LA TRAVESÍA, DE MODO QUE al Llegar a Puntarenas ya estaba lista. Astuto COMO ERA, Y PENSANDO EN EL ECO INTERNACIONAL DE 
SU LUCHA, PIENSO QUE HIZO OTRA COPIA (A MANO, PUES NO HABÍA MÁQUINAS DE ESCRIBIR) Y SE LA ENTREGÓ AL CAPITÁN J. W. LudWig, PARA QUE LA CIRCULARA en Panamá. Por cierto, en SU diario Personal, EL CÓNSUL INGLÉS RICHARD FARRER HABLA DE CIERTA COMPLICIDAD DE PARTE DEL CAPITÁN DEL COLUMBUS, QUE ERA LUDWIG EN ESE MOMENTO, Y NO JOHN M. Dow, QUiEn DESDE AGosto DE 1860 COMANDABA EL VAPOR GUATEMALA.

EN MI OPINIÓN, EL TEXTO DEL CORRESPONSAL F. W. R. TIENE UN GRAN VALOR HISTÓRICO, PUES APORTA ELEMENTOS QUE NI SIQUIERA DON CARLOS MeléndeZ Consignó en SU Detallado Libro sobre Montealegre. Puesto Que F. W. R. NO PResenció LOS HECHOS, ES LÓGICO SUPONER QUE QUIEN SE LOS RELATÓ FUE LUDWIG, Y ESTO PODRÍA A SU VEZ EXPLICAR ERRORES U OMISIONES, COMO LA MENCIÓN DEL SEÑOR AGUILAR, QUIZÁS CONFUNDIDO CON Manuel Argüello Mora, Que acompañaba a su tío don JuAnito en el Columbus.

Finalmente, más allá del valor del discurso, vendría a respaldar los hallazgos de Juan Durán Luzio acerca de la habilidad retórica de don Juanito. Es decir, ese discurso no podía haberlo redactado nadie más que él -otrora pudo haber contado con las plumas de Adolphe Marie y Emilio Segura, sus secretarios o amanuenses, que escribían muy bien, a menos que lo hubiera hecho Manuel Argüello Mora, notable escritor. Eso sí, tengo reservas acerca de esa habilidad de don Juanito, basado en tres cartas escritas por él cuando estaba preso y aislado, pocas horas antes de ser fusilado. Una de ellas, la de despedida de doña Inés Aguilar, su esposa, es la única que he tenido en mis manos y, obviamente, en ella se percibe que su caligrafía coincide por completo con la de la rúbrica de don Juanito. (Hilje, 2016)

Por su parte, la historiadora Carmen María Fallas Santana coincide "en la hipótesis de que el discurso no se publicó en el país por la derrota de los moristas y por el interés de la administración Montealegre de que no se conociera" (2014). Además, según Fallas Santana, existe coincidencia entre el contenido del párrafo del discurso que inicia con la expresión "no es el deseo de venganza lo que guía mis pasos" y

las declaraciones que hizo Mora, después de ser depuesto, [en el sentido] de que los que dieron el golpe de Estado fueron unos pocos traidores (entre los que siempre señaló a Lorenzo Salazar) y que pagarían por sus actos. Afirmaba que el resto de los involucrados habían actuado engañados por los traidores y que ante su eventual regreso, ellos podían estar tranquillos porque no iban a ser castigados. Lo anterior apunta a que el discurso efectivamente fue pronunciado por Mora. (Fallas Santana, 2014)

El historiador David Díaz Arias sostiene una posición similar a la de Fallas Santana, pues afirma que "este discurso tiene algunas ideas ya expuestas por Mora en su conocida proclama de enero de $1860^{8}$ y en un mensaje dirigido a los 'pueblos de Costa Rica' escrito en San Salvador en marzo de ese año9. Sin embargo, aunque parecido, no es ninguno de esos dos escritos" (Díaz Arias, 2014: 10). Y respecto a la pregunta de si realmente ese texto fue pronunciado por Mora Porras al llegar a Puntarenas, responde -en lo cual estoy de acuerdo- que "la pregunta queda abierta para los especialistas en Mora” (Díaz Arias, 2014: 10).

\section{Texto propuesto como traducción al castellano}

A partir de las dos versiones, publicadas en inglés en 1860 por The New York Times y el Panama Star and Herald, y tomando en consideración el léxico y el estilo discursivo presentes en los discursos, proclamas y mensajes presidenciales de Mora Porras, propongo el siguiente texto, como reconstrucción en castellano de lo que pudo ser la versión original de 1860:

JUAN RAFAEL MORA a sus conciudadanos.

Costarricenses:

Cuando en abril próximo pasado os hice saber las razones que me impidieron ayudaros a sacudir el yugo de vuestros opresores, no pude prever la magnitud que hoy alcanzan vuestros sufrimientos.

Nunca intenté abandonaros; solo os pedí esperar y trabajar por el bien del país, porque pensé que el tiempo restablecería la paz interna, sin que mi presencia fuera necesaria en esta República.

Desafortunadamente, estaba equivocado. Más de un año ha transcurrido desde los hechos del 14 de 
agosto. Entonces, vuestros gobernantes han tenido tiempo suficiente para hacer algo en beneficio del país, si hubieran sido capaces o lo hubieran querido.

Pero lejos de ello, el pasado año solo ha sido para Costa Rica una página de lágrimas y dolor. El pueblo castigado y oprimido, los sacerdotes de Cristo ultrajados y maltratados, los puertos cerrados al comercio, el Tesoro Nacional casi en bancarrota; en fin, vuestro país vendido, ofrecido indirectamente a los filibusteros. $\mathrm{Y}$ todo esto sin considerar la miseria general, el desaliento y la desesperanza que hacen más pesado vuestro trabajo y perturban vuestro reposo.

Ha llegado ya el día de que esto se acabe. Vuestros frecuentes llamamientos y el regreso a América Central del filibustero Walker, nos han movido, a los generales Cañas y Mora y a mí, a sacrificar nuestra tranquilidad por la independencia y el bienestar de Costa Rica.

¡Soldados, a las armas! Que la sangre que derramasteis en Nicaragua, Santa Rosa y el río San Juan no sea estéril. Y así venid a uniros con nosotros y no tendréis nada qué temer del porvenir.

No es el deseo de venganza lo que guía mis pasos; la clemencia se conforma mejor a mis sentimientos; así es que olvidaré los errores de aquellos que, engañados, prestaron sus servicios a los traidores; pero tened presente que seré inexorable con aquellos que, de hoy en adelante, no respondan al llamamiento de su agónico país, y haré que todo el rigor de la ley caiga sobre aquellos que presten cualquier tipo de ayuda al enemigo común.

¡A las armas, costarricenses! Apresuraos a uniros con nosotros y a romper vuestras cadenas al grito de ' $\mathrm{V}$ IVa Costa Rica'!

JUAN R. MORA.

Puntarenas, 17 de setiembre 1860. puede reconocer cierto grado de plausibilidad si se consideran los siguientes argumentos:

1.- $\quad$ El discurso tiene muchas similitudes con otros documentos atribuidos a Mora, en cuanto a forma y contenido, pero coincide principalmente con las proclamas que emitió en San Salvador en enero de 1860 y el 29 de marzo de 1860.

2.- $\quad$ Tales similitudes no se podrían explicar si el discurso se toma como apócrifo, salvo que su creador haya conocido profundamente las ideas y el estilo discursivo de Mora.

3.- La administración de Montealegre reconoció que Mora pronunció una proclama al desembarcar en Puntarenas y además conoció la versión del discurso publicada en inglés por el Panama Star and Herald.

4.- $\quad$ El Panama Star and Herald y The New York Times utilizaron dos fuentes distintas.

5.- Algunos de los detalles del relato publicado por The New York Times sobre el desembarco de Mora en Puntarenas, pueden ser indirectamente constatados triangulando con otras fuentes primarias, lo que permite atribuirle credibilidad a la narración.

6.- Existen dos versiones en inglés del discurso, que coinciden en cuanto al contenido, aunque no en la forma, lo que hace suponer que dos traductores trabajaron independientemente a partir de un mismo texto en español.

7.- $\quad$ Algunos expertos en el estudio de Mora y su época consideran como altamente posible que el discurso sea auténtico.

\section{Notas}

1. Ese era el nombre de Puntarenas, en inglés, en aquella época.

2. Es posible que "el señor Aguilar" era Manuel Aguilar Cueto, hermano de Inés Aguilar Cueto 
(esposa de Juan Rafael Mora Porras) e hijo de Manuel Aguilar Chacón (ex presidente de Costa Rica). Acerca de Manuel Aguilar Cueto el mismo Argüello Mora narra unos hechos ocurridos luego de la batalla de La Angostura: "Este valiente joven pertenecía a la guardia del Estado Mayor; lo sorprendieron durmiendo a la entrada del cuartel general y allí lo acribillaron a balazos; pero en vez de apuntarle al pecho o a la cabeza, se complacían con tirarle a las piernas y brazos, y no fue sino al cabo de horribles sufrimientos cuando el mismo desventurado joven se apoderó de un revólver, que arrancó a un soldado que estaba cerca de él, montó el arma, se la acercó a la boca, y todo concluyó. 'Bueno, bravo - gritaban unos soldados-; bien lo mereció el tal Manuel Argüello, más bien debían haberlo matado al nacer'. ¡Oh, y así por el estilo! Creían que el Manuel fusilado era yo" (Argüello Mora, 2011: 43-44). Según la Gaceta Oficial de Costa Rica del 25 de enero de 1860 el 23 de enero de 1860 partió de Puntarenas, con destino a los puertos de Centroamérica, el vapor Guatemala, con los siguientes pasajeros: “Alonso Gutiérrez, José Gabriel Segura, Rafael Chavarría, Manuel Aguilar, James Guidors, Federico Alvarado, doña Inés Aguilar y familia, don Miguel Mora, D. J. Ulloa, y doce en tránsito” (p. 2).

Pero podría tratarse de Mauro Aguilar Cueto, también hermano de doña Inés y colaborador de Mora Porras en sus planes de retomar el poder. La Gaceta Oficial de Costa Rica del 14 de diciembre de 1860 manifestó respecto a Mauro Aguilar: "Un mes después de haber anunciado pública y solemnemente que desistía de sus proyectos de invasión, vemos a D. Juan R. Mora ocupado ya en fraguar nuevamente planes. A fin de llevarlos a efecto con más facilidad, regresan al país, validas de la tolerancia del Gobierno, algunas personas de las que habían querido seguir la suerte de su Jefe, entre ellas D. M. Mora, y el ex Vicepresidente de la República, quienes habían salido voluntariamente, pero que se habían mezclado de un modo público en concitar enemigos contra el país, y en todos los preparativos de la frustrada invasión. Uno de los agentes revolucionarios que de este modo se internaron, fue D. Mauro Aguilar, hermano político de D. Juan R. Mora...” (p. 3).

Ahora bien, quizá The New York Times cometió un error y más bien quiso hacer mención de Manuel Argüello Mora, sobrino de Mora Porras. Argüello también venía en el Columbus de El Salvador y desembarcó junto con Mora Porras el 17 de setiembre de 1860 en Puntarenas.

3. El pasaje de Panamá a Puntarenas costaba 40 dólares estadounidenses y de Panamá a La Libertad, 75 dólares estadounidenses y el pago podía realizarse en oro estadounidense (Otis, 1861: 149).

4. El Capitán de Puerto era el coronel Salvador Mora, nombrado en 1860 en ese puesto por la administración de Montealegre. "Esto intrigó mucho a todas las gentes, tanto partidarios como opositores del régimen, pues era sabido que ese militar era tenido como apasionado morista, al que había apoyado en hora muy temprana, desde que se planteó la lucha que determinó la caída del doctor Castro y el ascenso al poder de don Juan Rafael. Algunos individuos, con un poco de visión, sospecharon que lo que se trataba de hacer era ponerle una trampa a don Juanito para hacerlo caer en las redes que ya estaban tendidas para su captura" (Meléndez Chaverri, 1968: 105).

5. Posiblemente existe un error en la fuente y el señor Santander de origen peruano quizá sea en realidad el chileno Ignacio Arancibia. O bien, se trata de José Tomás Santander, militar también chileno que apoyó a Mora Porras en los hechos de setiembre de 1860. Luego de estos Santander fue acusado formalmente y se le impusieron "los castigos que la ley establecía" (Meléndez Chaverri, 1968: 126). Manuel Argüello menciona dos veces a Santander en La trinchera: "Santander, chileno de muy buena familia, nos prestó sus conocimientos militares" (1899: 23) y agrega: “...el traidor, el General don Rafael Chavarría era ayudante de Cañas, y Santander, segundo del mismo" (1899: 25-26). También Argüello menciona a Santander en Elisa Delmar: “¡A las armas! -exclamó Santander, el segundo de Cañas, chileno de buena familia, valiente y buen mozo, a quien el destino condujo a nuestras playas en esa época. Al instante estuvo cada hombre en su puesto" (2007: 150).

6. Muchachos. Esta palabra aparece en castellano en el original.

7. El Daily Alta California, en su edición del 28 de octubre de 1860 , publicó una narración 
similar, aunque más concisa del desembarco de Mora en Puntarenas. Afirmó que la información la había tomado del Panama Star and Herald del 9 de octubre de 1860 y que este último a su vez la había recibido del corresponsal de ese periódico en la ciudad de Puntarenas, quien escribió su reporte con fecha 3 de octubre de 1860 (The Tragedy Execution of Ex-President Mora and General Canas, 1860).

La proclama de enero de 1860 se publicó en Costa Rica en la edición del 11 de febrero de 1860 de Nueva Era y en la Gaceta Oficial de Costa Rica de 11 de abril de 1860.

La proclama de 29 de marzo de 1860 se publicó en Costa Rica en Gaceta Oficial de Costa Rica de 11 de abril de 1860 .

\section{Bibliografía}

Argüello Mora, Manuel. 1899. La trinchera. San José: Imprenta y Librería Española M. v. de Lines.

Argüello Mora, Manuel. 2007. Elisa Delmar. Novela histórica. En Victoria Azofeifa Camacho, comp. Obras literarias e históricas. San José: Editorial Costa Rica: 143-160.

Argüello Mora, Manuel. 2011. Páginas de historia. En Iván Molina Jiménez, ed. Las primeras biografías de Juan Rafael Mora. San José: Editorial Costa Rica: 7-52.

Chomsky, Noam. 1957. Syntactic Structures. The Hague: Mouton Publishers.

Díaz Arias, David. 2014, 20 de julio. El discurso final de Juan Rafael Mora Porras. En La Nación, Sección Áncora: 10.

Durán Luzio, Juan, ed. 2011. Juan Rafael Mora Porras: primer ensayista costarricense, escritos selectos. San José: Imprenta Lara Segura.
End of the Revolution in Costa Rica. 1860, 31 de octubre. Sacramento Daily Union: 1.

Fallas Santana, Carmen María. 2014, 23 de setiembre. Correo electrónico enviado a Oriester Abarca Hernández.

Gaceta Oficial de Costa Rica. 1860, 25 de enero: 2.

Gaceta Oficial de Costa Rica. 1860, 11 de abril: 3-4.

Gaceta Oficial de Costa Rica. 1860, 13 de octubre: 3-4.

Gaceta Oficial de Costa Rica. 1860, 14 de diciembre: 3-4.

Hilje, Luko. 2016, 6 de marzo. Correo electrónico enviado a Oriester Abarca Hernández.

Meagher, Thomas Francias. 1923. Vacaciones en Costa Rica. Traducción de Ricardo Fernández Guardia. San José: Imprenta Trejos.

Meléndez Chaverri, Carlos. 1968. Dr. José María Montealegre: contribución al estudio del hombre y una época poco conocida de nuestra historia. San José: Academia de Geografía e Historia.

Montúfar, Lorenzo. 1887. Reseña histórica de Centroamérica. Tomo sétimo. Guatemala: Tipografía La Unión.

Mora Porras, Juan Rafael. 1860, 11 de febrero. El Presidente de la República a los pueblos de Costa Rica. En Nueva Era, año 1, número 14: 1 .

Otis, Fessenden Nott. 1861. Illustrated History of The Panama Railroad; together with a Traveler's Guide and Business Handbook for the Panama Railroad and 
Its Connections with Europe, The United States, The North and South Atlantic and Pacific Coasts, China, Australia, and Japan, by Sail and Steam. New York: Harper \& Brothers Publishers.

President Mora's Address. 1860, 27 de setiembre. Panama Star and Herald: n. d.

Sacramento Daily Union. 1860, 26 de octubre: 2.
The New York Times. 1860, 8 de octubre: 8.

The Tragedy - Execution of Ex-President Mora and General Canas. 1860, 28 de octubre. Daily Alta California: 1.

Vargas Araya, Armando. 2013. El lado oculto del Presidente Mora: Costa Rica versus el expansionismo esclavista de Estados Unidos. San José: Eduvisión.

\section{(c) $\Theta \Theta \Theta$}


